\title{
DARWIN: Demonstrator for an Adaptive and Reconfigurable Wireless IP Network ${ }^{\star}$
}

\author{
Guillem Femenias, Josep Lluis Ferrer, and Loren Carrasco \\ Departament de Matemàtiques i Informàtica, Universitat de les Illes Balears \\ Cra. de Valldemossa km. 7,5, Palma de Mallorca 07122, Spain \\ guillem.femenias@uib.es, dijjfg@tomir.uib.es, loren@ipc4.uib.es
}

\begin{abstract}
Project DARWIN is working towards the specification and demonstration of a flexible broadband WLAN radio access system based on an IP network platform. In a sense, DARWIN can be considered as an evolution of IEEE 802.11 and HIPERLAN/2. This paper presents the main innovative ideas behind this project, that can be summarized as: a flexible network architecture providing extended range ad-hoc and infrastructure modes of operation, enhanced air interface (low-density parity check (LDPC) codes, adaptive modulation, smart antennas combined with space-time block codes), enhanced wireless link and data link control functions, and an enhanced security architecture.
\end{abstract}

\section{Introduction}

Within the Spanish ICT (Information and Communications Technology) initiative, project DARWIN is working towards the definition and demonstration of a flexible broadband WLAN radio access system based on an IP network platform. DARWIN systems are intended to provide short range, wireless access to multi-media services over IP, operating in the $5 \mathrm{GHz}$ band and providing bit rates up to $100 \mathrm{Mbps}$. In a sense, DARWIN can be considered as an evolution of IEEE 802.11 and HIPERLAN/2 and, as such, the main innovative ideas behind it can be summarized as:

- a flexible network architecture providing extended range ad-hoc and infrastructure modes of operation;

- enhanced air interface, through the use of low-density parity check (LDPC) codes, adaptive modulation algorithms, smart antennas at both transmitter and receiver combined with space-time block codes, etc;

- enhanced wireless and data link control functions that fulfil the requirements on Quality of Service (QoS) and IP support dictated by upper layers; and

- an enhanced security architecture.

In the first phase of the project specifications of the different system layers have been generated. In a second phase the functionality of the system is being

* This work has been supported in part by the Ministerio de Educación y Ciencia, Spain, and FEDER (Fondo Europeo de Desarrollo Regional), under grant TIC20010287. 


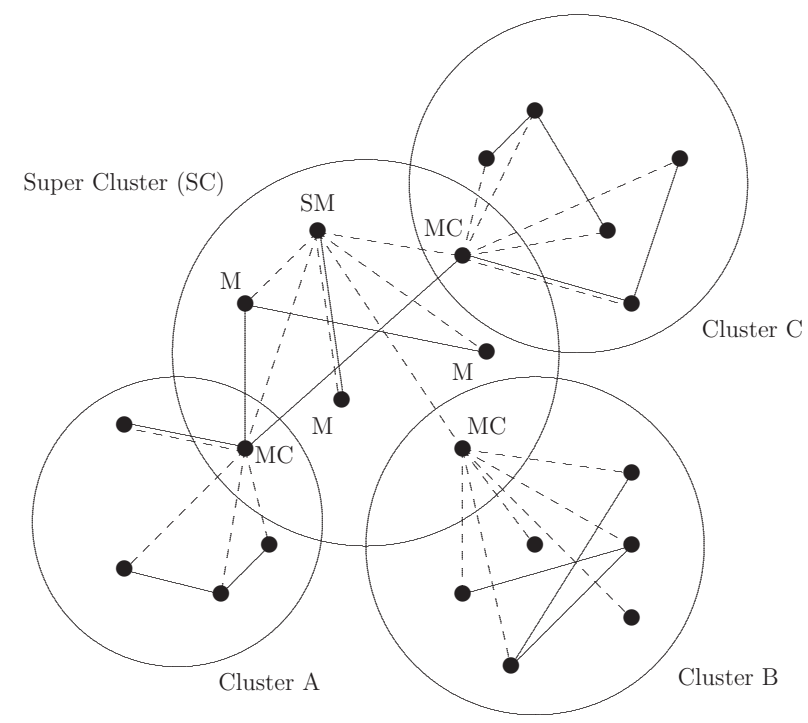

Fig. 1. DARWIN subnet

tested through the use of simulation tools. The third phase of the project is intended to provide as a result a prototype implementation of the system.

\section{Flexible Network Architecture}

One of the main features of DARWIN is its flexibility in network configuration. A DARWIN network can be defined as a group of DARWIN devices which have in common the same NET-ID. Within a DARWIN network we can define a subnet as a set of synchronized DARWIN devices using the same carrier frequency. A subnet is created when a Darwin Super Master (DSM) starts to generate valid Broadcast Control CHannel (BCCH), and allows other DARWIN devices to associate with the network. All devices of a subnet shall be synchronized to the frequency chosen by the DSM, and access the channel using the MAC frame structure dictated by the DSM. The selection of the DSM is dynamic, and seamless switching of the DSM responsibility from one DSM-capable wireless terminal to another is possible. Apart from the DSM, all the other devices in a subnet belong to one of the following hierarchical categories: Darwin Master (DM), Darwin Master of a Cluster (DMC) or Darwin Slave (DS). A DM is a device that is directly associated to the DSM and does not have any other device associated with it. A DMC is a DMC-capable device that is associated to the DSM and has other devices associated with it. A DS is a device that is associated to the DSM through the intermediation of a DMC. A DMC together with its associated DSs constitute a cluster and the DSM, the DMs and the DMCs of a subnet constitute a super-cluster.

The control plane of DARWIN is centralized for all general features. That is, only the DSM, either directly or through the mediation of a DMC, can instruct 


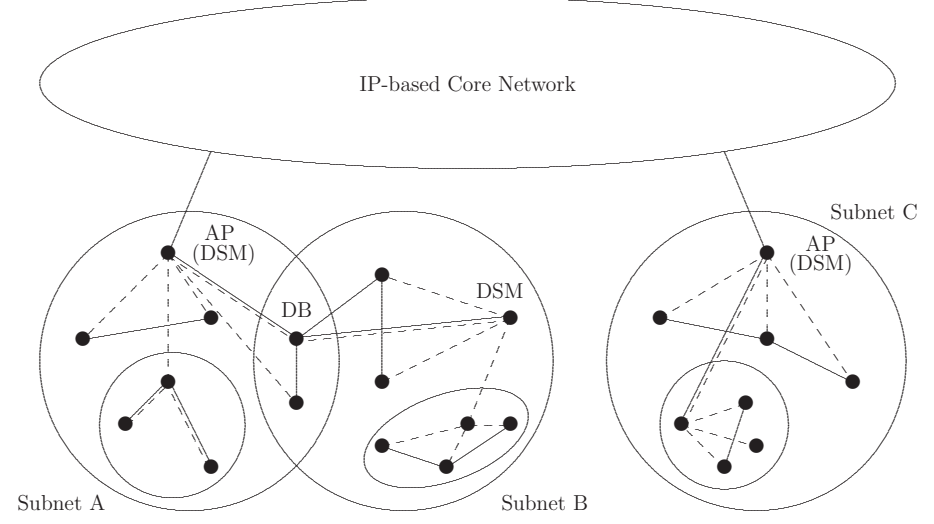

Fig. 2. General architecture of a DARWIN network

a DARWIN terminal to do something. However, distributed control is also made possible for some ad-hoc features by introducing logical control channels, which can be used for direct exchange of control messages between terminals. In the user plane, DARWIN makes extensive use of Direct Link (DiL) user connections. Direct Mode is used, provided that two wireless terminals can reach each other directly. A link quality calibration process helps to track the connectivity between any two devices by measuring the associated RF link quality. The DSM (or DMC) is used as a user data relay for a pair of terminals, provided that they cannot reach each other directly. Even this user data relaying is performed during the DiL phase between the wireless terminals and the DSM (or DMC).

Apart from providing connectivity between wireless terminals (ad-hoc network), DARWIN can also provide connectivity of wireless terminals to access points (AP) that bridge traffic to wired IP networks (infrastructure based network). In this case, the role of DSM will be played by the AP and DARWIN will work as a seamless extension of other networks, so wired IP network nodes will see DARWIN nodes as other network nodes.

In order to increase the capacity or the coverage area of a single subnet, multiple subnets operating on different frequencies can be deployed. Each subnet is under the control of its own DSM and works independently of the other subnets. Dynamic Frequency Selection (DFS) is used to enable dynamic selection of the RF channel. Different subnets can be interconnected either by a fixed network through the APs (infrastructure based network), or by DARWIN Bridges (DB) (ad-hoc network). Potentially any DB-capable DM device in a subnet can be configured as a bridge to another subnet.

\section{Physical Layer Enhancements}

DARWIN physical layer [1] is very similar to that adopted by IEEE 802.11 and HIPERLAN/2. Parameters like channel raster, OFDM subcarrier spacing, cyclic prefix duration, burst structure, ... coincide with those used in IEEE 802.11 and 

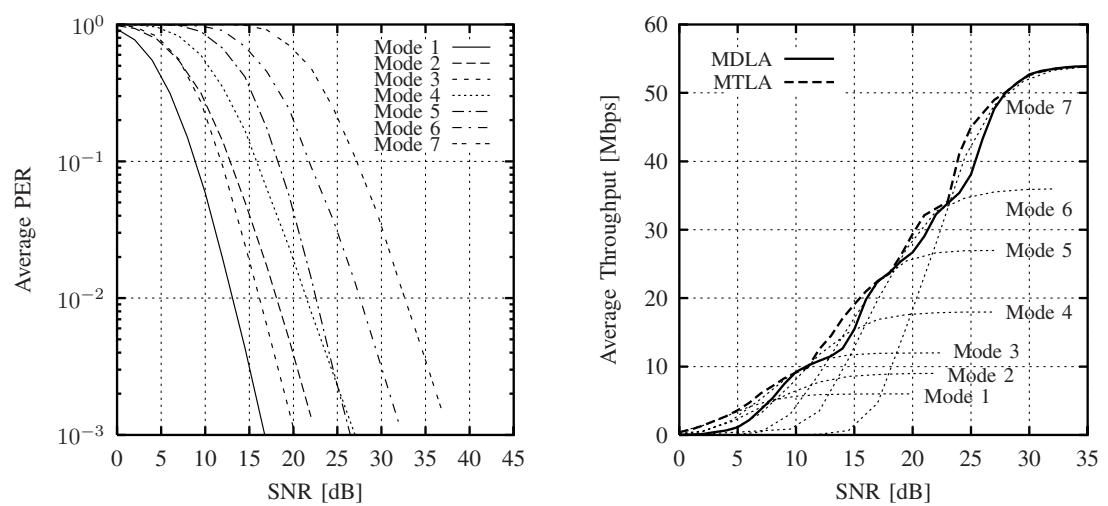

(a)
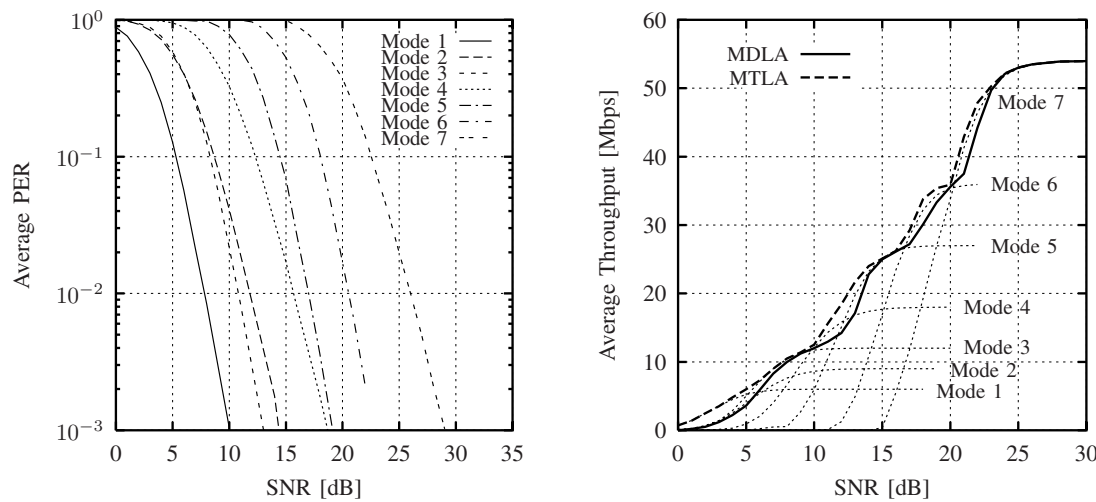

(b)

Fig. 3. Average PER and average throughput of (a) conventional (punctured $\left(R_{c}=\right.$ $1 / 2, K=7$ ) convolutional code) and (b) enhanced (punctured $(864,432)$ irregular LDPC code combined with Alamouti's STBC) systems. It has been assumed the transmission of 54-bytes packets over ETSI BRAN Channel A. Two link adaption algorithms have been implemented: minimum delay (MDLA) and maximum throughput (MTLA).

HIPERLAN/2. However, in order to fulfil the requirements of QoS dictated by upper layers, the physical layer of DARWIN incorporates a series of enhancements with respect to IEEE 802.11 and HIPERLAN/2 systems. In particular, some of the techniques that are being investigated within DARWIN project are:

- use of adaptive coding strategies based on low-density parity check codes;

- use of adaptive modulation schemes;

- use of smart antennas at both transmitter and receiver combined with spacetime block codes.

Preliminary results (see Fig. 3) show that these techniques can provide performance improvements in terms of packet error rate (PER) and throughput of the system. By providing a more reliable link, these enhancements result in a 


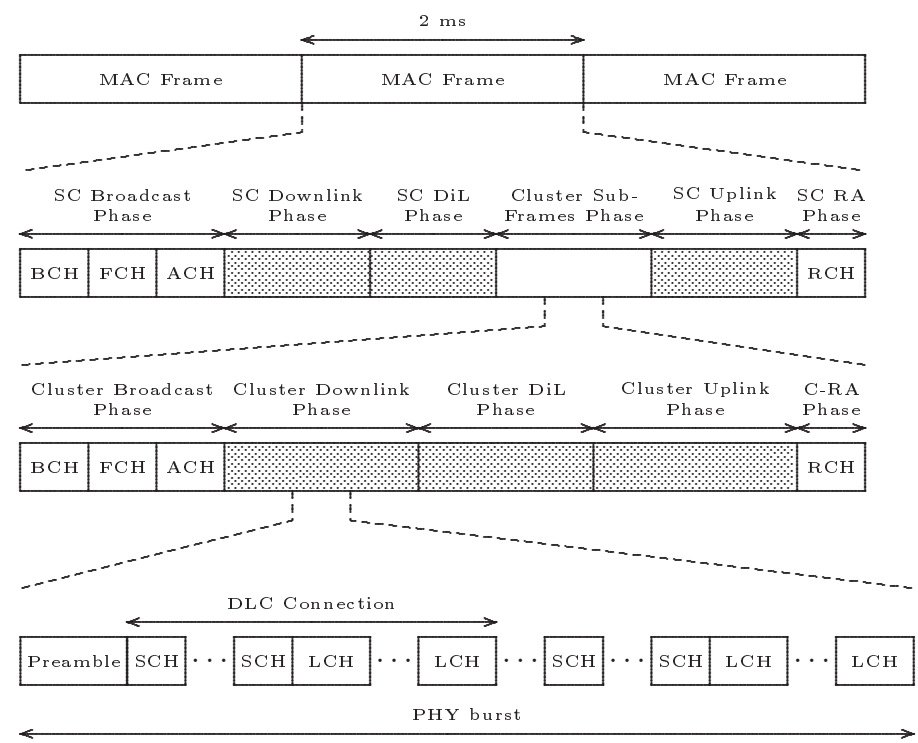

Fig. 4. Basic DARWIN frame structure (non-sectorized antennas).

significant improvement of the link control functions and, at the same time, contribute to one of the main objectives of DARWIN project, i. e. the consecution of an IP-based flexible broadband WLAN radio access system with QoS support.

\section{Data Link Control Layer Enhancements}

The DLC of DARWIN consists of three functional entities:

- Radio Link Control (RLC), responsible for the exchange of control functions' information between DSMs and DMs (or DMCs) and between DMCs and DSs. Supported DARWIN RLC control functions are: association control, radio resource control, DLC control, security management, QoS control, address management and configuration management.

- Error Control (EC), when applied to a user connection, is responsible for transmission error detection and recovery, and is based on ARQ.

- Medium Access Control (MAC), responsible for the implementation of DARWIN MAC protocol.

The DARWIN MAC protocol [2] is based on a dynamic TDMA/TDD scheme and its flexibility is, also, one of the main features of DARWIN project. Transmissions in downlink, uplink and direct link, are structured on the air interface as a sequence of MAC frames, each one of $2 \mathrm{~ms}$ duration. Six major variable-length phases can be identified in a typical DARWIN MAC frame as follows:

- Super Cluster Broadcast phase, in which control information is broadcast from the DSM to all the terminals in the super cluster. 
- Super Cluster Downlink phase, reserved for user control information and data transmission from the DSM to the terminals in the super cluster.

- Super Cluster Direct Link phase, reserved for user control and data direct transmissions between two terminals associated with the DSM and operating in direct mode.

- Cluster Sub-frames phase, in which DARWIN MCs are allocated a variableduration time slot that is also divided in five phases: cluster broadcast phase, cluster downlink phase, cluster direct link phase, cluster uplink phase and cluster random access phase.

- Super Cluster Uplink phase, reserved for user control information and data transmission from the terminals in the super cluster to the DSM.

- Super Cluster Random Access phase, consisting of one or more random channels, in which random access is allowed for transmission of control information from DMs or DMCs to the DSM.

The DARWIN MAC structure is able to extend the coverage of a DSM providing a 'relay' function for terminals that are outside the range of a DSM. The gain in communication range is paid with a degradation in system capacity. Planning strategies that result in an acceptable trade-off between these parameters are now being investigated, as well as powerful inter-layer scheduling structures that achieve a coupling between IP and DLC, providing QoS support.

\section{$5 \quad$ Security Services}

DARWIN incorporates all the basic security services, with a high degree of flexibility, allowing to negotiate services and algorithms to be used [3]. As a clearly differential element regarding HIPERLAN/2 and IEEE 802.11, DARWIN allows the use of public key infrastructure for authentication services. In relation to key exchange, besides Diffie-Hellman and pre-shared key, DARWIN establishes an scheme based on RSA. For confidentiality service DARWIN uses strong algorithms and secure key generation processes, based on the SSL model. DARWIN provides stream ciphers and block ciphers, and stablishes separated keys for both directions of communication. Finally, the integrity service is not implemented in HIPERLAN/2, and in IEEE 802.11 is linked to the confidentiality service. In DARWIN approach, integrity and confidentiality are independent services.

\section{References}

[1] DARWIN TR WG1.1 V1.0. Physical (PHY) layer, 2002.

[2] DARWIN TR WG2.1 V1.0. DARWIN MAC layer specification, 2003.

[3] DARWIN TR WG3.1 V1.0. DARWIN security services, 2003. 